\title{
Multi-Objective Boundary Tracking Method in Grayscale Image
}

\author{
Shengyuan $Y_{A N G}{ }^{a}$, Shanshan $X^{\mathrm{b} *}$, Xiaoyun $Z E N G^{c}$ and Yuanyuan PAN ${ }^{d}$ \\ College of Information Engineering, Xiangtan University, China

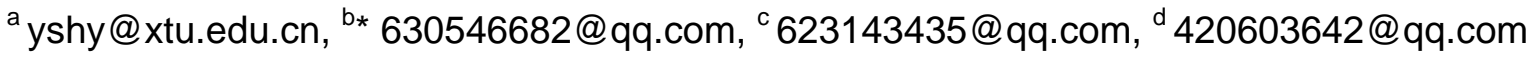

Keywords: grayscale image; multi-objective; gradient map; boundary tracking; seed point

\begin{abstract}
As for complex grayscale image, a kind of adaptive boundary tracking method for multi-objective is proposed in this paper. First of all, the grayscale image with noise is processed by Gaussian filter. Secondly, the image gradient after filtering is calculated. According to the geometric characteristics of ridge formed by edge points in gradient map, the new definition of seed points is put forward, which ensures the seed points in the seed point set must be ridge points, namely, edge points, and that there is at least one point in the edge points of the objectives belongs to the seed point set. Starting from the seed points, the boundary of all objectives can be tracked. The numerical experiments show that the method of this paper can adaptively track the multi-objective boundary in the complex grayscale image, and has a certain noise resistance and relatively strong robustness.
\end{abstract}

\section{Introduction}

Boundary tracking in image processing refers to starting from a boundary point in the image and searching for the next boundary point according to a certain identification standards to track the objective boundary in the image. Through boundary tracking, discontinuous boundary points can be converted into meaningful objective boundary information for practical applications such as license plate recognition, and face recognition.

There are many scholars studying upon the objective's boundary tracking in image and its application ${ }^{[1-6]}$. For example, boundary tracking based on binary images ${ }^{[1-4]}$. For binary images, it is assumed that there is only one objective with a closed boundary in the image and the boundary of the objective can be tracked according to the method of boundary searching by 4 neighborhoods or 8 neighborhoods. However, for some images, this method might lead to different results by choosing different starting points. For sure, for a non-binary image, the image may be binary processed by setting a threshold, but the given threshold might affect the accuracy of the objective boundary obtained via tracking. Freeman chain code representation ${ }^{[6]}$ has improved efficiency, but it needs to judge the direction of 8 neighborhoods with a considerable amount of calculation and the problem of missing tracking would rise when there are crossover conditions at the boundary. These binary image boundary tracking methods are more suitable for boundary tracking of single-objective images, but not for multi-objective boundary tracking, and cannot handle holes in images.

For the boundary tracking and application of multi-level grayscale image ${ }^{[7-14]}$, the determination of the starting search point, the boundary identification standards and the selection of search criteria are different from the boundary tracking algorithm based on the binary image. Generally, it is firstly to calculate the gradient map of the image by differential operator, and then to find the pixel point with the largest gradient as a starting search point, and then to find the second boundary point from the 8 neighborhoods of the starting point. The search ends when the gradient value of the newly found boundary point is less than a certain threshold. This method cannot effectively track boundary when there are multiple objectives in an image.

For a complex grayscale image with multiple objectives, this paper analyzes the gradient map of the filtered image and finds that the three-dimensional geometric ridge formed by the gradient map is aligned with the image objective boundary. If a point for each ridge could be located, it is possible to take those points as the seed points to extract the whole ridge line by using the boundary point tracking method and further outline the objective boundary line accordingly. Based on this 
consideration, this paper presents an adaptive multi-objective boundary tracking method for multi-objective boundary tracking in complex grayscale images.

\section{Definition of the seed point}

The two-dimensional Gaussian first derivative could be used as the filter to polish the grayscale image. The expression of the two-dimensional Gaussian function is as follows:

$$
G(x, y)=\frac{1}{2 \pi \sigma^{2}} e^{-\frac{x^{2}+y^{2}}{2 \sigma^{2}}}
$$

The symbol $\sigma$ is Gaussian filter parameter. Fig. 1 shows the vessel and its local gradient data.

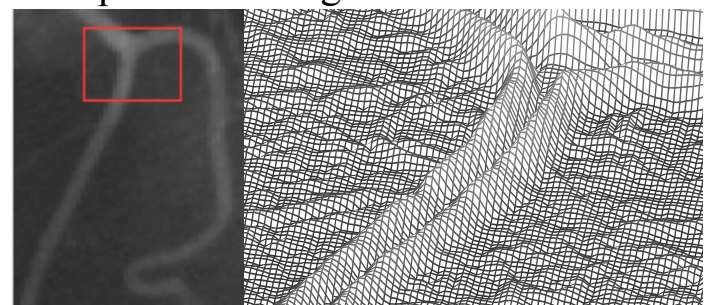

Figure 1.vessel and its local gradient data

From the local gradient map in Fig. 1, it can be seen that the grayscale in the grayscale image changes fiercely is the objective boundary, which is corresponded to the ridge in the gradient map with large gradient value, where the grayscale changes smoothly is consistent with the flat area in the gradient map with small gradient value.

Intuitively, if the points with large gradient values are connected in sequence, the obtained ridge curve is the objective boundary in the image. For the boundary tracking of single-objective image, the point with the largest gradient value must be on the ridge as the boundary point of the image. This point can be called the seed point. Starting from the seed point, it is easy to extract the objective boundary. However, for a complex grayscale image with multiple objectives, only the boundary of a certain objective can be obtained from the point with the largest gradient value, and the boundaries of other objectives will be missed. Therefore, it is necessary to obtain the seed points for each boundary so that all of boundaries can be effectively tracked. Therefore, for a multi-objective complex image, the point with the largest gradient cannot be simply taken as a seed point. It is necessary to provide a new method for obtaining seed points that facilitates tracking all boundaries.

According to the gradient map, the acquired seed points must be on the ridge-line, but it is not necessary to use all the ridge-line points as seed points. We only need to make sure that all acquired seed points in the seed point set is on the boundary line, and each boundary line has at least one point in the seed point set. In this way, all boundary lines can be tracked from the seed points.

Therefore, if such a ridge map meets one of the six situations shown in Fig. 2, point $\mathrm{C}$ can be used as the boundary point. According to the characteristics of the ridge, only the gradient of the other two points on the boundary within the neighborhood of point $\mathrm{C}$ may be greater than the gradient of point C. 


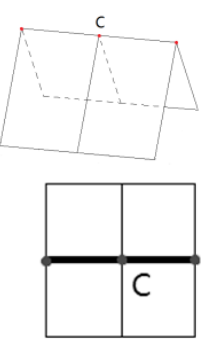

(a)

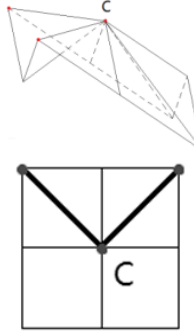

(e)

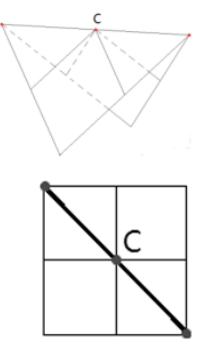

(b)

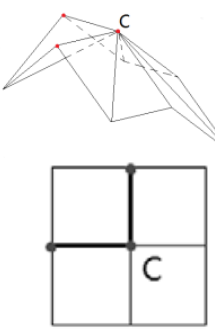

(f)

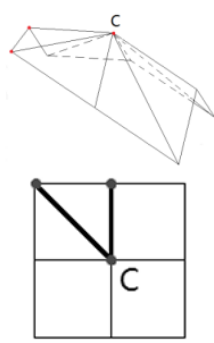

(c)

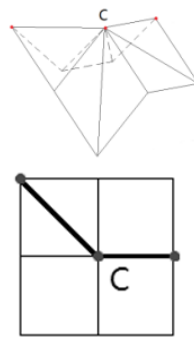

(g)

Figure 2. Illustration of field at boundary point $\mathrm{C}$

It can be seen from the Fig. 1(b) that the place where the grayscale changes smoothly also presents the situation in Fig. 2, but the gradient in this case is very small. In order to eliminate this false boundary, a threshold value $\theta$ will be set. The gradient of the boundary point must be greater than the threshold $\theta$. The value of threshold $\theta$ is between 0 and 1 . There is a reasonable range for the threshold $\theta$ in every image. If the threshold is smaller than this range, some boundary points with lower gradient values might be included in the seed set, which may track redundant boundary; if it is greater than this range, some real boundary points couldn't meet the definition of the seed point, which results in the situation of missing tracking. Only when the threshold $\theta$ takes a reasonable range, it can be ensured that there is at least one seed point on each boundary, and the seed point only exists with the boundary points.

Based on the above analysis, the definition of the seed point is given:

Definition (seed point) In the gradient map, for a center point $C$, if the gradient value of 2 points in the $3 \times 3$ neighborhood of center point $C$ is greater than the gradient value of point $C$, and the gradient value of point $\mathrm{C}$ is greater than the given threshold $\theta$, then the point $\mathrm{C}$ could be seen as the seed point.

According to the definition of the seed point, after the gradient values of the gradient map are normalized, the number of seed points decreases with the increase of the threshold $\theta$. As shown in Fig. 3 , the three images in the second row are the seed point images when the threshold $\theta$ is $0.3,0.2$, and 0.3 respectively, and the three images in the third row are the seed point images when the threshold $\theta$ is $0.15,0.1$, and 0.15 , respectively.

It can be seen that the number of seed points in the third row is more than that in the second row in Fig. 3, indicating that the smaller the threshold $\theta$ is, the larger the number of seed points. However, it isn't the case that the small threshold $\theta$ the better. It can be seen from the third row of Fig. 3 that there are a lot of seed points with false boundaries in the acquired seed point map. 


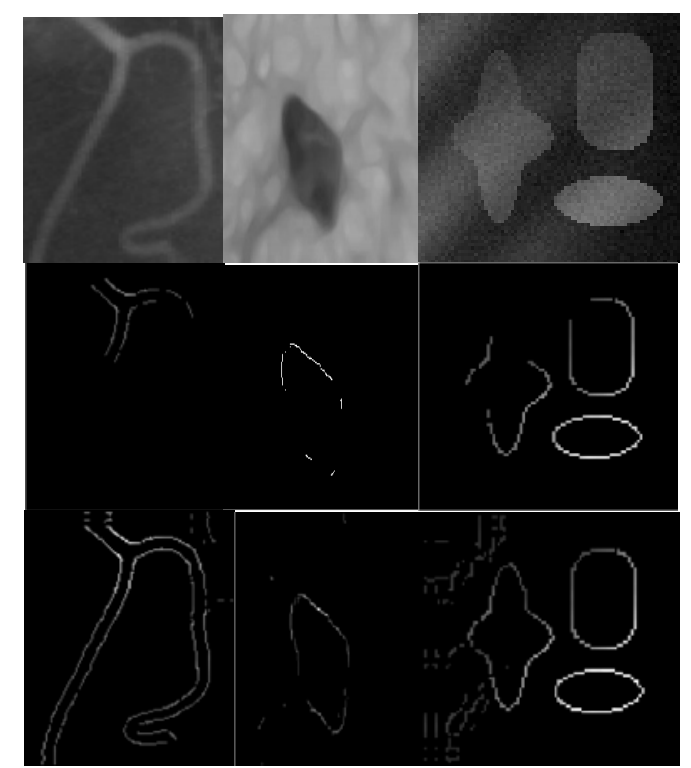

Figure 3. Original image and different threshold seed points

\section{Multi-objective boundary tracking algorithm analysis and design}

Considering that in the complex image, the boundary might gradually merges with the background, and the corresponding gradient value is also very small, this paper believes that it does not belong to the boundary point. To simplify the subsequent search operation, a small threshold $\alpha$ could be set. When the gradient value is smaller than $\alpha$, the corresponding gradient value of these points is set to 0 during the preprocessing, and the value of the threshold $\alpha$ falls in the interval of $[0, \theta)$. For the objective boundary and the background is not clear image, the threshold $\alpha$ can be set to 0 to avoid missing the objective boundary with unclear grayscale change. If the value of the threshold $\alpha$ is greater than $\theta$, the boundary tracked by the algorithm could only be scattered points whose gradient values are greater than $\alpha$.

All seed points are detected and form a seed set. Scanning through the seed point set, for each seed point taken out from the seed point set, the point with the largest gradient value in its 8 neighboring points (as shown in Fig. 4) is identified after a clockwise scanning to be taken as the next boundary point. If the boundary point is also a seed point, it will be deleted from the seed point set. The current boundary point is noted as $\mathrm{C}$, and the previous boundary point is noted as $\mathrm{P}$. In order to improve the efficiency, three gradient points in front of the PC direction, as shown in Fig.5, can be selected during the detection process to determine the next boundary point. When the located point has been identified as a boundary point, or the gradient is 0 , or it is already a boundary point of the entire image, detection is performed from another direction of the seed point until the point found also fit the above conditions. At this point, this round of detection ends. Then, another seed point is taken out of the seed set for further detection, until the seed point set becomes empty and the boundary point detection thereby ends. 


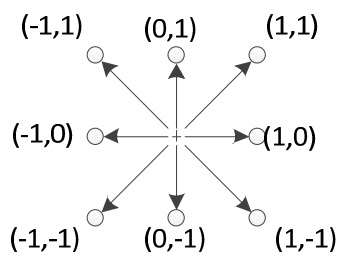

Figure 4. Eight neighbor points of seed points

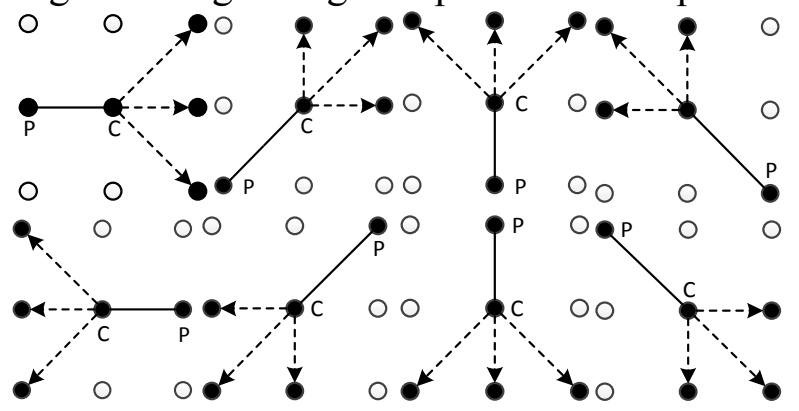

Figure 5. Retrieving boundary tracking schematic eight directions

For multi-objective boundary tracking in complex grayscale images, based on the above analysis, a multi-objective boundary tracking algorithm can be given as follows:

Step1. Use a Gaussian filter to filter grayscale images.

Step2. Use the boundary detection operator to calculate the gradient of the filtered image;

Step3. Set a threshold $\theta$ and traverse the gradient of each pixel. When the gradient is greater than $\theta$, further check whether it is a seed point. If it is a seed point, add the point to the seed point set $\Omega$;

Step4. Traverse the seed point set $\Omega$. If $\Omega$ is an empty set, the traverse ends. If $\Omega$ is not empty, then take a seed point from $\Omega$, which can be regarded as seed point i, then perform the following steps:

S1. Mark the seed point $i$ as a boundary point, and note the boundary point as P; clockwise detect the 8 points of the seed point $\mathrm{i}$ in the neighborhood (see Fig. 4) to find the point with the largest gradient, and note it as point $\mathrm{C}$. At the same time, point $\mathrm{C}$ is additionally saved as temporary point;

S2. If point $C$ is a boundary point or an image boundary point, perform $\mathrm{S} 6$; otherwise perform $\mathrm{S} 3$;

S3. Mark point $C$ as a boundary point. If point $C$ is a seed point, remove it from seed point set $\Omega$.

$\mathrm{S} 4$. Along the direction of $\mathrm{P}$ pointing to $\mathrm{C}$, to detect the gradient value of the first three points (as shown in Fig. 5), select the point where the gradient is the largest, and note the point as T;

S5. If $\mathrm{T}$ is a boundary point or the gradient value is 0 or an image boundary point, perform S6; otherwise, point $\mathrm{C}$ is marked as point $\mathrm{P}$, point $\mathrm{T}$ is marked as point $\mathrm{C}$, and perform $\mathrm{S} 3$;

S6. Mark seed point $\mathrm{i}$ as point $\mathrm{C}$ and mark temp point as point $\mathrm{P}$;

$\mathrm{S} 7$. Along the direction of $\mathrm{P}$ pointing to $\mathrm{C}$, to detect the gradient value of the first three points (as shown in Fig. 5), select the point where the gradient is the largest, and note the point as T;

$\mathrm{S} 8$. If $\mathrm{T}$ is a boundary point or the gradient value is 0 or an image boundary point, perform $\mathrm{S} 4$; otherwise, point $\mathrm{C}$ is noted as point $\mathrm{P}$, point $\mathrm{T}$ is noted as point $\mathrm{C}$, and performs $\mathrm{S}$.

S9. Mark the point $\mathrm{C}$ as the boundary point. If it is a seed point, remove it from seed point set $\Omega$.

S10. Return to perform S7 and moving object appears.

Fig. 6 shows the main flowchart of the algorithm in this paper. 


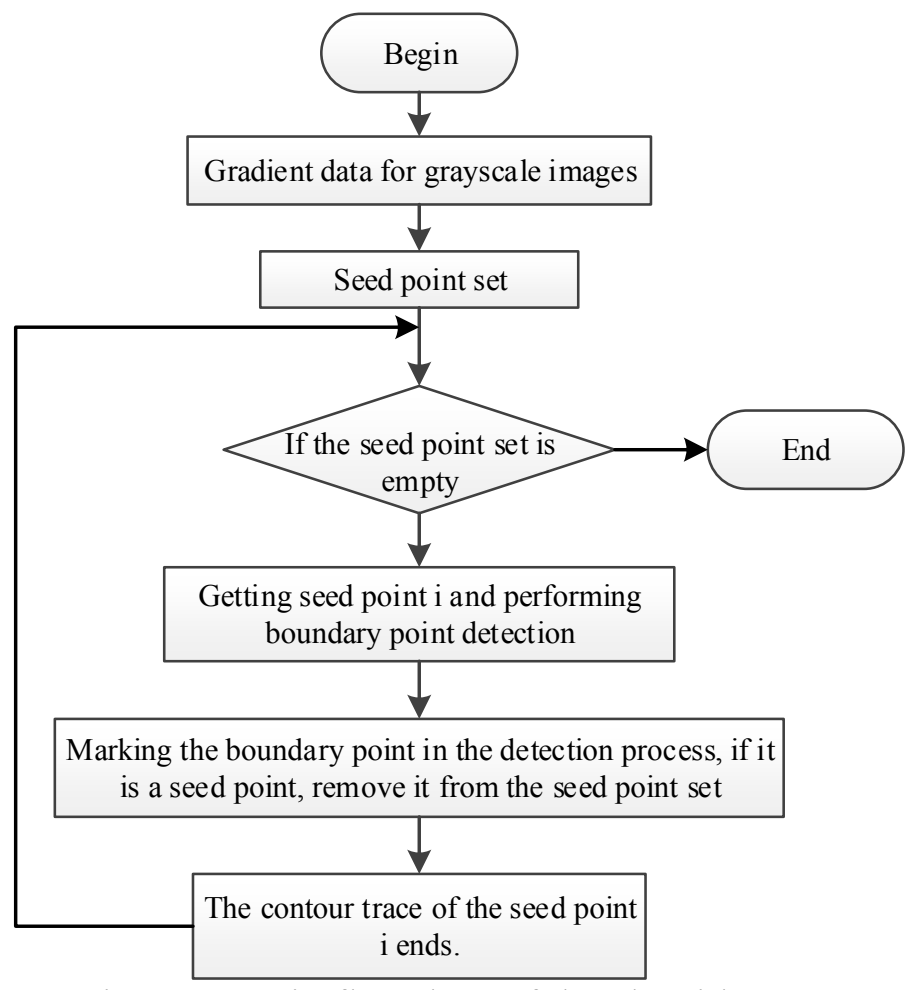

Figure 6. main flowchart of the algorithm

\section{Numerical experiments and analysis}

In this section, boundary tracking experiments are performed using the different grayscale images of the boundary tracking algorithm to verify the effectiveness of the proposed algorithm.

There are two images in the first row in Fig. 7 contain multiple objectives and Gaussian white noise. Threshold $\theta$ and threshold $\alpha$ are $0.3,0.2$ and $0.3,0.05$, respectively. The two images in the second row in Fig. 7 are the corresponding results obtained by being tracked with the proposed algorithm. It is good at tracking the boundary information of the geometrical objectives in the image and well deal with noise, indicating that this method have good resistance to noise.

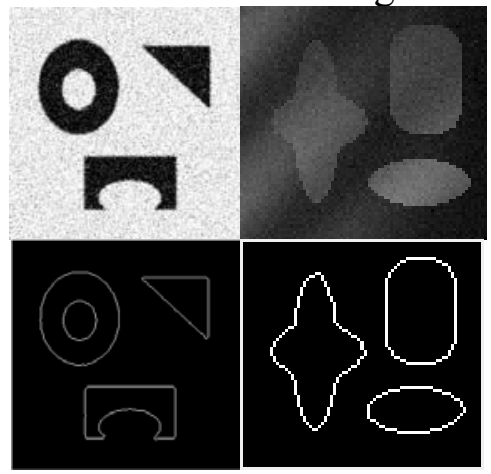

Figure 7. Boundary tracking of the new method in this paper

The two images in the second row of Fig. 8 and the two images in the third column of Fig. 9 are the corresponding boundary information obtained by being tracked with the algorithm. The second row threshold $\theta$ and the threshold $\alpha$ in Fig. 8 are 0.15, 0 and 0.3, 0.05, respectively, and the third column threshold $\theta$ and the threshold $\alpha$ in Fig. 9 are $0.13,0$ and $0.13,0.02$, respectively. Images in the second column of Fig. 9 uses the modified Canny method [13] to obtain the results extracted from the boundary. It can be seen from the comparison that the new method can extract complete and continuous boundaries. 


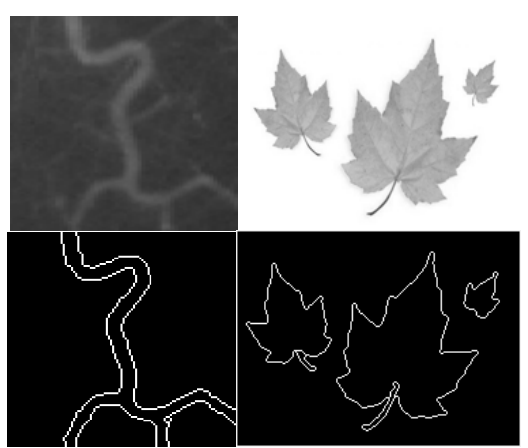

Figure 8 . Boundary tracking results for two complex grayscale images

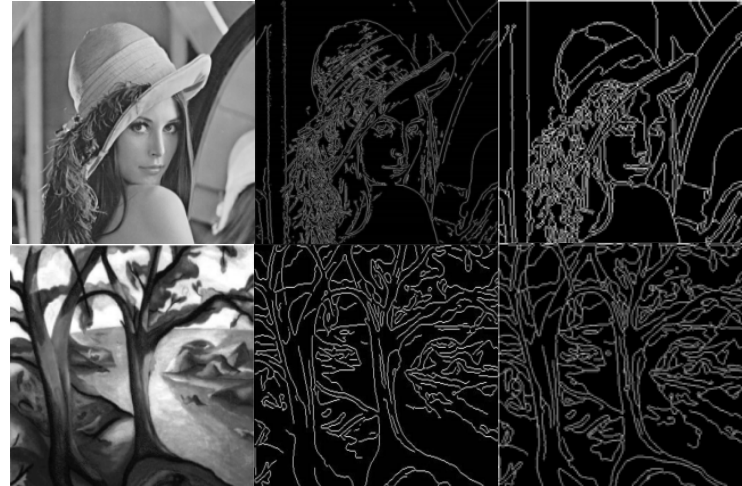

Figure 9. Boundary trace results of two grayscale images

The images of the column a in Fig.10 are two grayscale images, which are: blood vessel image and skin lesion images. The column $b$ in Fig. 10 are the results of the boundary extraction using the Canny method ${ }^{[14]}$. The column $\mathrm{c}$ in Fig. 10 are the results of boundary tracking using the method proposed in this paper, Threshold $\theta$ and threshold $\alpha$ are $0.15,0$ and $0.025,0$ respectively. For the treatment of the blood vessel with non-occluded contour image in the first row of Fig. 10, the proposed method can completely extract all of contours of the blood vessel. At the bottom of the image, Canny method cannot effectively extract the boundary of the blood vessel, but the proposed method can reasonably detect the boundary information. For the treatment of the skin lesion image, the Canny method fails to extract the complete boundary information of the objective due to the inconspicuous change in the grayscale of the contour of the lesion. The boundary line appears to be disconnected and split somewhere. However, the proposed method can not only track the complete boundary, but also no false boundary.

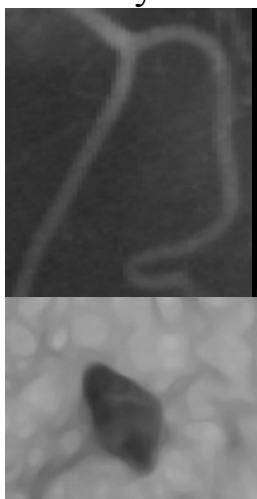

(a)

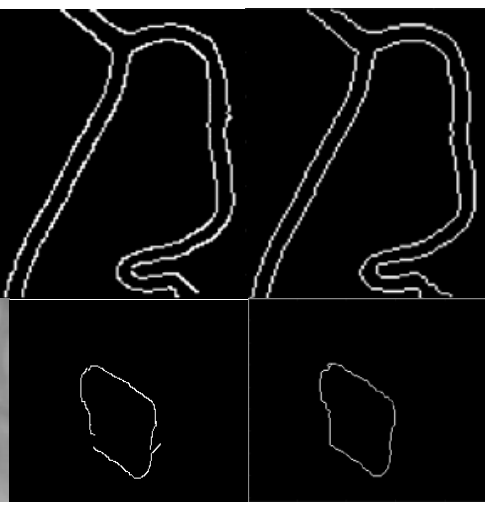

(b) (c)

Figure 10. Boundary trace results of two grayscale images

\section{Conclusions}

This paper presents an adaptive multi-objective boundary tracking method for multi-objective boundary tracking problem in complex grayscale images. The method proposes the definition of new seed point. The definition of seed point ensures that the seed point must be a ridge point, that is, the boundary point, and the boundary point of each objective must belong to the seed point set. 
Experimental results show that this method can effectively track the boundary information of multi-objective.

\section{Acknowledgement}

This work is supported by National Natural Science Foundation of China (No.11571293) and open Project of KeyLaboratory of Hu'nan Province in the 12th Five-Year Plan(No.2015IM05), all support is gratefully acknowledged.

\section{References}

[1] M Ren, J Yang, H Sun. Tracing boundary contours in a binary image. Image and vision computing, 2002,20(2):125-131

[2] D Sun, Y Liu. A New Contour Tracing Algorithm in Eight-Connected Binary Images. Proceedings of Third International Joint Conference on Computational Science \& Optimization, IEEE Conference Publications, 2010, 1:249-253.

[3] X Zhou, Y Chen, W Hu. Tree Traversal Binary Image Boundary Tracking Algorithm Based on Cross-Point. Computer Applications and Software, 2014, 31(2):230-232. (in Chinese)

[4] W Chen, Q Guo, W Fan. et. A Rapid Algorithm on Tracing Edge of Binary Image and Its Application. Engineering of Surveying and Mapping, 2014, 23(12): 63-66. (in Chinese)

[5] J Zheng, J Zhang, N Zhao. Extraction of Blood Vessel in Lung CT Images Based on Tracking Algorithm. Computer Engineering and Applications, 2007, 43(32):204-206. (in Chinese)

[6] J Wang, W Song, L Zhao. et. Application of Improved Freeman Chain Code in Edge Tracking and Straight Line Extraction. Journal of Signal Processing, 2014, 30(4):422-430. (in Chinese)

[7] K Gu, W Fu, Y Dong. et. Rapid Pupil Detection Based on Improved Contour Tracking. Infrared Technology, 2017, 39(6):574-578. (in Chinese)

[8] Q Li, H Wen, Y Qu. et. Application of Edge Tracking Algorithm to Multiple Paper Defects Segmentation. China Pulp \& Paper, 2017, 36(8):41-45. (in Chinese)

[9] T Wei, L Yuan. Highly real-time blind sidewalk recognition algorithm based on boundary tracking. Opto-Electronic Engineering, 2017, 44(7):676-684. (in Chinese)

[10] E Chen, J Wang, L Qi. Novel multiscale edge tracking algorithm for edge detection. Application Research of Computers, 2015, 32(12):3801-3804. (in Chinese)

[11] X Huang, Y Su, Y Liu. Iteratively parsing contour fragments for object detection. Neuro computing, 2016, 175(PA): 585-598.

[12] S Badsha, H Arof, N Mokhtar.etc, Segmentation of overlapping Cryptosporidium and Giardia (oo)cysts using bidirectional contour tracing. Biomedical Signal Processing \& Control, 2015, 18:161-168.

[13] C Deng, G Wang, X Yang. Image edge detection algorithm based on improved Canny operator. International Conference on Intelligent Systems Design \& Applications, 2013, 38(14):623-628.

[14] X Wu, W Yu, Y Ma. A New Improved Canny Image Edge Detection Algorithm. Imaging Science and Photochemistry. 2016, 34 (1): 116-121. (in Chinese) 